\title{
Psychological Treatment for Rheumatoid Arthritis Works: Now We Need to Know What Elements Are Most Effective and for Whom
}

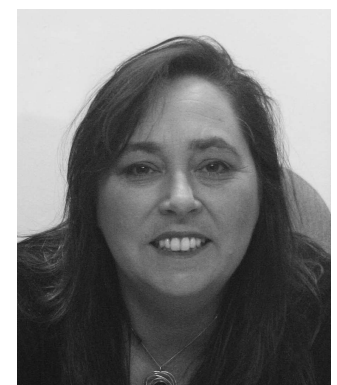

In this issue of The Journal, Shadick and colleagues ${ }^{1}$ conduct a proof-of-concept study investigating the efficacy of a novel psychosocial intervention for patients with rheumatoid arthritis (RA): Internal Family Systems-based Psychotherapy (IFS). IFS has not previously been the subject of randomized controlled trials (RCT) and therefore its potential efficacy is unknown. Group differences favoring the IFS group over a group receiving routine care were found for pain severity and physical function at post-treatment, and for joint function (self-assessed), depression, and self-compassion at 1 year followup. Hence, this trial has established proof of concept for the potential efficacy of IFS in RA. But what is IFS and how is it related to existing evidence-based treatments?

Internal family systems therapy was developed and articulated by Schwartz ${ }^{2}$, and his book indicates that much of the influence for IFS has come from work in family therapy ${ }^{3,4}$. However, IFS is not a form of family therapy per se, because patients were seen in groups without other members of their family present. Shadick, et $a l^{1}$ described the processes: (a) IFS "teaches patients to attend to and interact mindfully" with their internal experiences; and (b) IFS "recruits self-compassion and conceptualizes parts of a person as subpersonalities, in which they are encouraged to engage in a dialogue with polarized thinking." According to Shadick, et $a l^{1}$, patients are encouraged to identify situations with their concomitant thoughts and emotions. Patients are then asked to identify the origin of these experiences, allowing them to change their response and identify compromise positions to allow them to behave differently. From this description, it is clear that IFS shares overlap with numerous other models of psychotherapy, many of which have been previously used in RA.

It is important to determine the potential overlap with existing, evidence-based treatments, to identify the effective components of intervention for patients with RA. Over a decade ago, Astin and colleagues ${ }^{5}$ metaanalyzed 25 RCT of psychological interventions for patients with RA and concluded that there was evidence for the efficacy of psychological interventions over and above standard care in patients with RA. That result has recently been replicated, including studies on both RA and osteoarthritis in a qualitative review of the literature ${ }^{6}$. However, importantly, we still know very little about which particular therapies are more effective and whether different patients benefit from different approaches. Williams and colleagues found good metaanalytic data to support the use of cognitive-behavioral therapy (CBT) for chronic pain ${ }^{7}$. CBT aims to challenge patient beliefs and help them to change their behavior. Hence, in RA, patients are encouraged to adopt a more optimistic (but realistic) attitude toward their illness and to learn strategies to help themselves pace their activities better, to be able to achieve important life goals ${ }^{8}$. Is IFS similar to CBT? How does it differ?

CBT for RA consists of cognitive therapy and behavioral therapy. Cognitive therapy aims to help patients distance themselves from their thoughts and identify thoughts and their relationships with emotions and behavior ${ }^{8}$. A range of strategies are introduced to help patients develop alternative interpretations of situations that lead to negative emotions, such as determining whether beliefs are realistic and helpful, and constructing more helpful ways to view a given situation. Cognitive therapy also helps patients to identify their underlying beliefs and to identify situations in which these beliefs may have developed ${ }^{8}$. These strategies are, in fact, quite similar to those described by Shadick and colleagues $^{1}$. That is, both approaches encourage distance between the person's internal experiences (i.e., thoughts and feelings) and help patients to identify relationships between these experiences. IFS encourages dialogue between a more compassionate voice and the critical voices experienced by patients, while cognitive therapy achieves change through a combination of challenging the accuracy of beliefs and their helpfulness. Hence, the major difference

See Systems-based psychotherapeutic intervention on outcomes in RA, page 1831

Personal non-commercial use only. The Journal of Rheumatology Copyright (C) 2013. All rights reserved. 
between cognitive therapy and IFS appears to be that IFS does not explicitly challenge the accuracy of beliefs.

The behavioral component of CBT for RA focuses more on trying to help patients adopt a more balanced approach to activity $^{8}$. That is, patients explicitly set goals and learn skills in pacing themselves to establish a better balance between rest and exercise. Other strategies, such as relaxation, are also often included, to give patients additional skills in achieving rest. From the description of IFS, it is less clear that these behavioral strategies are the focus of IFS, although some examples used by Shadick, et al indicate that patients may have been encouraged to find a better balance between rest and exercise (e.g., "a patient would become more aware of the need to compromise between collapsing and stoically ignoring his or her pain") ${ }^{1}$. Why is the overlap between CBT and IFS important?

CBT is the best-researched psychological therapy for RA in particular ${ }^{5,6}$ and for pain in general ${ }^{7}$. However, the vast majority of trials compare CBT to standard care or a wait list design. While these designs help us to know whether CBT is more effective than no psychological intervention, they do not indicate whether CBT is better than other viable treatments. Further, the effect sizes from metaanalyses and reviews suggest that while $\mathrm{CBT}$ is effective, the effect sizes are small to moderate, hence there is considerable room for improvement ${ }^{5,6,7}$. Understanding the commonalities of effective interventions will help us to identify the effective components of treatment.

For example, there is only 1 trial in the literature that has attempted to dismantle CBT to determine the most effective components of treatment. Sharpe and Schrieber ${ }^{8}$ compared a CBT program previously found to be effective for patients with $\mathrm{RA}^{9,10,11}$ with the purely behavioral components (including goal setting, pacing, and relaxation) and the purely cognitive components (including cognitive challenging and distraction). There was no evidence that behavioral strategies added anything to the cognitive strategies administered alone. That is, cognitive therapy alone outperformed at least 1 of the comparator treatments on $3 / 7$ outcomes. These results suggest that it may be the changes in attitudes and beliefs that are fundamental to the changes observed in CBT for patients with RA. It is interesting that, while taking a different approach to change a person's internal dialogue and reduce self-criticism, IFS also targets these constructs.

We need to encourage the conduct of other large-scale RCT that compare different psychosocial interventions to each other. One further reason that it is important to compare active treatments is so that we can choose the most appropriate intervention for a particular patient. It is likely (if not inevitable) that different patients may benefit from different approaches. Without studies that directly compare more than 1 intervention and compare subgroups of patients, it is impossible to know in what way one might most effectively tailor interventions toward particular patients.

In the only study in the literature to take this approach, Zautra and colleagues ${ }^{12}$ compared CBT with mindfulness-based meditation. Mindfulness-based meditation is sometimes considered the "third wave" of CBT. Mindfulness differs from CBT in that it does not aim to change the content of people's beliefs (as in cognitive therapy), but rather people's relationship with their thoughts and other internal experiences. That is, in mindfulness, patients are encouraged to become objective observers of their own thoughts and experiences but to respond to these with curiosity and not react to them. Notably, IFS also aims to help patients develop a more mindful relationship with their thoughts. In the Zautra and colleagues ${ }^{12}$ trial, they did not find any evidence for the superiority of either CBT or mindfulness for the entire group of patients with RA. However, for people with RA who have a history of clinical depression, mindfulness was more effective than CBT. These results are similar to results that have consistently found that mindfulness meditation is particularly well supported for people with a history of depression (3 or more episodes) who are currently not depressed ${ }^{13,14}$. From this study, it appears that RA patients with recurrent depression may similarly benefit more from mindfulness than from traditional CBT.

At present, it is clear that CBT is the most strongly empirically supported intervention for patients with $\mathrm{RA}^{5,6,7}$. However, we urgently need research that investigates the relative efficacy of different psychosocial approaches. The contribution of Shadick, et $a l^{1}$ is important because it shows proof of concept for IFS, a novel intervention. Nonetheless, a single trial does not prove the approach effective. Until more research is conducted with approaches such as IFS, CBT will remain the psychological treatment of choice for patients with RA. More RCT comparing different psychosocial treatments for patients with RA are needed to determine the most effective treatment and to understand which patients will benefit most from which approaches.

\section{LOUISE SHARPE, PhD,}

Professor of Clinical Psychology, School of Psychology,

Brennan MacCallum Building A18,

The University of Sydney,

Sydney, NSW Australia

LS is supported by a National Health and Medical Research Council Senior Research Fellowship.

Address correspondence to L. Sharpe. E-mail: louises@psych.usyd.edu.au

\section{REFERENCES}

1. Shadick NA, Sowell NF, Frits ML, Hoffman SM, Hartz SA, Booth FD, et al. A randomized controlled trial of an internal family systems-based psychotherapeutic intervention on outcomes in rheumatoid arthritis: a proof of concept study. J Rheumatol 2013;40:1831-41. 
2. Schwartz R, ed. Internal family systems therapy. New York: Guilford Press; 1995.

3. Minuchin S, ed. Families and family therapy. Cambridge, MA: Harvard University Press; 1974.

4. Bowen M, ed. Family therapy in clinical practice. Lantham, MD: Rowman \& Littlefield Publishers Inc.; 2004.

5. Astin JA, Becknew W, Soken K, Hochberg M, Berman B. Psychological interventions for rheumatoid arthritis: a meta-analysis of randomized controlled trials. Arthritis Care Res 2002;47:291-302.

6. Keefe FJ, Smith SJ, Buffington AL, Gibson J, Studts JL, Caldwell DS. Recent advances and future directions in the biopsychosocial assessment and treatment of arthritis. J Consult Clin Psychol 2002;70:640-55.

7. Williams AC, Eccleston C, Morley S. Psychological therapies for the management of chronic pain (excluding headache) in adults. Cochrane Database Syst Rev 2012:14:1-108.

8. Sharpe L, Schrieber L. A blind, randomised controlled trial of cognitive versus behavioural versus cognitive-behavioural treatment for patients with rheumatoid arthritis. Psychother Psychosom 2012;81:145-52.

9. Sharpe L, Sensky T, Timberlake N, Ryan B, Brewin CR, Allard S. A blind, randomized, controlled trial of cognitive-behavioural intervention for patients with recent onset rheumatoid arthritis: preventing psychological and physical morbidity. Pain 2001;89:275-83.
10. Sharpe L, Sensky T, Timberlake N, Ryan B, Allard S. Long-term efficacy of a cognitive behavioural treatment from a randomized controlled trial for patients recently diagnosed with rheumatoid arthritis. Rheumatology 2003;42:435-41.

11. Sharpe L, Allard S, Sensky T. Five year follow-up of a cognitive-behavioural intervention for patients with rheumatoid arthritis: effects on health care utilisation. Arthritis Rheum 2008;15:311-6.

12. Zautra AJ, Davis MC, Reich JW, Nicassario P, Tennen H, Finan P, et al. Comparison of cognitive behavioral and mindfulness meditation interventions on adaptation to rheumatoid arthritis for patients with and without history of recurrent depression. J Consult Clin Psychol 2008;76:408-21.

13. Segal ZV, Williams JMG, Teasdale JD. Mindfulness-based cognitive therapy for depression, 2nd edition. New York: Guilford Press; 2013.

14. Piet J, Hougaard E. The effect of mindfulness-based cognitive therapy for prevention of relapse in recurrent major depressive disorder: a systematic review and meta-analysis. Clin Psychol Rev 2011;31:1032-40.

J Rheumatol 2013;40:1788-90; doi:10.3899/jrheum.131043 